\title{
LA EFICIENCIA DEL MERCADO, MÁS ALLÁ DE LOS MODELOS MATEMÁTICOS
}

\author{
DANIEL LAHOUD*
}

Fecha de recepción: 23 de julio de 2012.

Fecha de aceptación: 19 de diciembre de 2012.

\section{I \\ INTRODUCCIÓN}

Los años sesenta son el momento en el cual se desarrollaron los estudios académicos dirigidos a plantear la Hipótesis de la Eficiencia de los Mercados(en inglés Efficient Markets Hypotesis, $\mathrm{EMH}$ ), sin embargo, el primer antecedente es atribuido a Louis Bachelier quien en su tesis, Teoría de la Especulación, estableció un modelo matemático para estudiar los comportamientos del mercado, en el año 1900. Como se indica, es un modelo de comportamiento, por lo que considera que el mercado tiene un comportamiento definido, que es propio como objeto de investigación de la Psicología Social, es decir del mundo del conductismo, y por tanto Positivista.

Hay quienes piensan que el mercado bursátil es un «casino» y quizá el más señero representante de esa tesis es J.M. Keynes. A pesar de ser él mismo un asaz especulador, afirma en su Teoría General, ${ }^{1}$ cuando se refiere al mercado como el juego infantil de las sillas y la música que de tanto en tanto se detiene, para dejar a alguien siempre de pié, por tanto perdiendo el juego en cuestión.

Algunos otros creen que el estudio, el análisis y los conocimientos de los mercados otorgan una ventaja y consideran que los mercados de inversión pueden ser predichos con anticipación.

* Economista, Historiador y Profesor de la Universidad Católica Andrés BelloCEDICE de Venezuela y Profesor de la UCV en Caracas.

${ }^{1}$ Keynes (1981), pp. 145-146. 
Por supuesto, también hay quienes consideran que el comportamiento del mercado es azaroso y que sigue lo que llaman una caminata aleatoria. Afirmando que si es así, entonces los mercados funcionan bien como sistemas de ahorro para el público y de financiación para las empresas, pero los sustentadores de esta teorías hacen la salvedad que este es el comportamiento de los mercados desarrollados o globalizados.

La teoría de la EMH se basa en que el precio de los títulos negociados en bolsa tienen incorporada toda la información que es conocida por los inversionistas, intermediarios y en general por todos los actores del mercado, lo que significa que han descontado en su apreciación las expectativas que ellos guardan sobre el futuro. Por tanto, es imposible lograr superar los resultados del mercado, y la única manera de batirlo es, contando con suerte o teniendo acceso a información privilegiada. ${ }^{2}$ Dado que las noticias que afectan a un título se deberían reflejar en tiempo real en el precio de las acciones, el proceso por el cual ocurre esto es aleatorio y no puede conocerse anticipadamente.

Es importante dejar claro que la teoría de la EMH acepta la posibilidad de que existan comportamientos irracionales, debido a que los inversionistas generalmente sobrerreaccionan o infrarreaccionan ante las noticias de una manera aleatoria. Por lo cual, el mercado es quien se comporta de una manera irracional durante un plazo largo, y los eventos como depresiones de la economía y crash del mercado, son compatibles con la EMH siempre y cuando no sean predecibles.

La EMH, es y ha sido una teoría bastante discutida. Hay quienes la validan pero también quienes la refutan, su fundamentación está en que los mercados tienen memoria en el largo plazo y que existen ineficiencias del mercado que dan lugar a beneficios extraordinarios, pero estos beneficios son temporales.

${ }^{2}$ El criterio generalmente aceptado de información privilegiada implica que aquellas personas o instituciones que estén vinculadas de manera directa o indirecta, en las actividades relacionadas con los mercados de valores deben garantizar la confidencialidad en los datos relevantes, y especialmente, impedir que alguien disponga de esta información, que se denomina privilegiada y que el gobierno debe procurar garantizar la transparencia de los mercados y castigar judicialmente el uso de esta información privilegiada y la manipulación de los mercados. 
El presente documento, será una revisión del estado del arte en el «mainstream»y un planteamiento de la eficiencia dinámica, como alternativa para entender el mercado financiero.

\section{II \\ DESARROLLO TEÓRICO}

\section{La Eficiencia del Mercado}

De acuerdo a lo expuesto en la teoría, la eficiencia del mercado está relacionada con el proceso de formación de los precios de las inversiones en su propio mercado, y tiene implícitas las premisas: 1) que las rentabilidades esperadas deben estar en equilibrio y en función del riesgo inherente, es decir, deben reflejar un precio justo, entendiendo esta justeza como el reflejo del valor presente de los flujos de caja descontados, es decir aquel que refleje la información relevante; 2) que las variaciones en los precios deben ser independientes, es decir, con una correlación entre ellos a lo largo del tiempo, igual a cero. Este es el fundamento para que los precios obedezcan a un comportamiento aleatorio y además, que las rentabilidades históricas no tendrán ninguna influencia sobre las rentabilidades futuras.

El primer trabajo, el de Bachelier, se inspiraba en el llamado movimiento browniano, también caminata aleatoria, o deambular del marino ebrio, que son trabajos que se desarrollaron en el siglo XIX con la intención de estimar el movimiento de un grano de polen en un medio líquido. Es decir, un fenómeno de laboratorio, que ni siquiera se da en la naturaleza donde no actúa ninguna mente humana, incluso el movimiento de un grano que no es un ser vivo, sino un corpúsculo floral. Tratando de extrapolar este fenómeno con el mundo de las ideas que vive en un mercado, como es el mercado financiero.

Desde los años sesenta se ha propuesto la teoría de la EMH, que se ha encargado del estudio del comportamiento de las cotizaciones bursátiles, para verificar que los precios de las acciones reflejan la mejor y más completa información disponible. Según ésta teoría, si el mercado es eficiente, los inversionistas no pueden 
vencer en forma constante a un índice simple de precios de acciones (el cual representa el mercado).

Cootner (1962: 24-45), expresó el concepto de eficiencia del mercado de la manera siguiente:

Aunque los compradores o vendedores individuales pueden actuar con ignorancia; tomados como un todo, los precios de tal mercado reflejarán la mejor evaluación del conocimiento disponible en cada momento. Si un grupo sustancial de inversores pensara que los precios son demasiado bajos, sus compras forzarían los precios al alza. La inversa también sería cierta para los compradores... En este ámbito, las únicas variaciones de precio que podrían ocurrir, serían las resultantes de nueva información. Dado que no hay razón para esperar que la información no sea aleatoria en su aparición, las variaciones de período a período, del precio de un valor, deberían ser movimientos aleatorios, estadísticamente independientes entre sí. El nivel de los precios, bajo estas condiciones, describiría lo que los estadísticos denominan un recorrido aleatorio, y los físicos un movimiento browniano.

La afirmación de Cootner es interesante y merece que el analista se detenga a interpretarla a la luz de la lógica teórica. Es cierto, que los inversionistas pueden actuar con ignorancia frente al mercado, y si lo hacen, es porque probablemente fueron exitosos en la aplicación de un decisión similar en una situación anterior, y están experimentando de nuevo en la creencia de que van a obtener el mismo resultado, o están actuando por primera vez, creyendo que esa es la actitud que se debe asumir en la inversión financiera. Pero, no todos los inversionistas actúan de esta manera, hay algunos que actúan de acuerdo a estrategias, que los han convertido en exitosos y probablemente estos, actúen como agentes, de personas que tienen menos conocimiento y no pueden alcanzar toda la información que es necesaria para establecer esas estrategias. Pero, acumular conocimiento y disponer de información no es la única condición del éxito, incluso algunos que pueden actuar de una manera totalmente ignorante, alcanzan un beneficio mejor, la única diferencia quizá, es que el último es incapaz de recrear el fenómeno y aprender de él. Eso sí, el estudioso puede perder tantos recursos monetarios propios o ajenos 
en el error, que lo conduciría a no poder recuperarse o perder su credibilidad ante sus «principales» en la relación de agencia. Entonces es importante definir que el gerente de una cartera actúa como un empresario, en un ambiente de cambios constantes e impredecibles, y para subsistir debe tener una especial creatividad así como una habilidad adaptativa que lo hace altamente especializado. Esa perspicacia es mucho más compleja en los mercados de inversión debido a que su ambiente de incertidumbre está acrecentado, frente al mundo de los mercados de bienes de orden cercano al consumo.

Los precios pueden parecer bajos, sin embargo eso no ocasionaría per se el alza. Deben presentarse toda una gama de fenómenos que sean analizados por los inversionistas para que sus expectativas cambien haciéndose positivas y conduciendo al mercado a un proceso de alza sostenida. Incluso, esta alza puede ocasionarse sin que signifique un cambio en la expectativa del mercado, porque quienes estén en el mercado con posiciones cortas, deciden cubrir dichas posiciones temporalmente. Si eso conduce a una situación en la que los compradores superen a los vendedores, se experimentará, al menos de manera momentánea, una recuperación en los precios, sin embargo, como la expectativa de largo plazo no ha cambiado, el mercado retornará a su condición bajista; por supuesto, condiciones similares, pero en sentido contrario llevarían a una reacción alcista de los precios. Pero, aunque el comportamiento es azaroso, nunca podría describir, como en la física, una caminata aleatoria, porque el movimiento no es, ni puede ser discreto, y sus probabilidades no seguirían nunca el comportamiento de una función. Porque no existen diferenciales en la medición de los precios en una bolsa, porque tampoco las cantidades pueden ser discretas, no puede negociarse fracciones menores a una acción, y tampoco pueden darse variaciones en los precios que sean menores a la menor fracción de dinero en una economía.

Al poco tiempo, Samuelson (1965) afirmaba que el mercado no necesariamente se comporta como una caminata aleatoria, pero si afirma que el comportamiento de los precios no puede ser pronosticado, debido a que no existe una relación clara entre el comportamiento de los precios pasados y el comportamiento de los 
precios en el presente. Esa premisa es perfectamente cierta, porque al estar en un mercado de capitales, el precio de una acción, por ejemplo, no tiene ninguna relación con el pasado. La empresa que está representada por la acción puede haber sido exitosa, y se dirige a la quiebra, o al contrario puede haber estado en situación de atraso y se dirige a obtener ganancias extraordinarias. Si el mercado puede detectar esas situaciones, el precio estará determinado por lo que ocurrirá, no por lo que ocurrió. Por eso ningún modelo que utilice el pasado puede servir para estimar el presente de la valoración de la inversión.

Ahora el trabajo de Samuelson incluyó otros supuestos adicionales: 1) que todas las personas están en la misma disposición de información, sin que esto represente costos adicionales y además 2) que todas las personas tienen el mismo horizonte de inversión y las mismas expectativas. Estos dos supuestos son definitivamente falsos.

Para que las personas posean la misma información, ésta debe ser asequible en el mismo momento para todos, eso es imposible, y aún teniéndola no podría ser analizada con el mismo marco referencial por los participantes, por diversas razones:

1. no tenemos el mismo conocimiento o

2. escoger de la información la parte que nos parece relevante,

3. sencillamente no poseemos la misma información,

4. la procesamos de una manera distinta

Eso es lo que lleva en la realidad a los vendedores a considerar que el precio debería bajar y por eso venden. Al mismo tiempo los compradores a estimar que el precio debería subir y por eso compran. Entonces, de una manera natural, todos tenemos expectativas diversas y además el tiempo en el que creemos que ese proceso se va a dar, debería ser distinto y eminentemente subjetivo. Si existiesen expectativas iguales, nadie podría negociar, porque sencillamente el comprador no conseguiría vendedores, o el vendedor no conseguiría compradores. El tiempo también es relevante, porque si todos tenemos el mismo horizonte de inversión todas las compras se harían en el mismo momento y todas las ventas se harían de la misma manera, pero de nuevo si eso 
fuese así, el vendedor no conseguiría compradores y el comprador no conseguiría vendedores, por lo que no habría mercado. Eso lleva las valoraciones al plano de lo subjetivo, porque si tenemos la misma información y un conocimiento y expectativas diversas, entonces la única manera es que las valoraciones sean subjetivas y los tiempos también lo sean. Lo que es perfectamente compatible con el primer supuesto que se analizó antes, es decir que los precios no tienen que ver con el pasado, y que se relacionan con el valor presente de lo que ocurrirá en el futuro y esas expectativas no pueden ser nunca iguales.

Cómo afirma Cootner, puede haber movimientos alcistas o bajistas, pero esto no son producto de una información igualmente distribuida y un conocimiento igualmente distribuido. Ambas condiciones son desigualmente distribuidas en cualquier sociedad, independientemente del grado de desarrollo de la misma. Además que sería necesario establecer un "precio justo objetivo» para que se establezca situaciones de irracionalidad, por la sobrerreacción o por la infrarreacción. Sin embargo, las valoraciones son subjetivas y por tanto no existe un precio justo, sino infinidad de precios justos, tantos como personas estimen las ganancias futuras de una determinada inversión.

Siguiendo en el objetivo de definir el mercado eficiente, Fama (1970: 383-417), supone que los precios reflejan toda la información disponible y orientan a sus partícipes acerca de cómo deben ser sus decisiones de inversión:

El mercado ideal es aquel, en el que los precios proporcionan indicaciones exactas para la asignación de recursos. Esto es, un mercado en que las empresas puedan llevar a cabo decisiones de producción e inversión, y en el que los inversores puedan elegir entre los títulos valores que representan la propiedad de la empresa, bajo el supuesto de que los precios de los mismos, reflejan por completo, en todo momento, toda la información disponible.

La definición que plantea, interpreta la situación de un mercado ideal, y ciertamente todo mercado es un mecanismo de información, hasta aquellos que están altamente regulados están en la disposición de proporcionar datos para la asignación de 
recursos, eso sí mientras menos regulados son, mejores son los precios para servir como señales para la asignación de los recursos. En el caso de un mercado de inversión, los precios permiten dirigir más eficientemente los recursos financieros. Naturalmente, si los precios se forman en un mercado intervenido, esa es una condición que puede conducir a errores en la decisión, y se puede ejemplificar, cada vez que un banco central modifica las tasas de interés creando una información que se añade como información nueva a la preexistente en el mercado.

Pocos años después, Jacquillat y Solnik (1975:22), definen un mercado eficiente como aquel mercado «que incluye instantáneamente las consecuencias de los acontecimientos pasados y refleja en forma precisa las expectativas expresadas sobre los acontecimientos futuros. Así, el precio de un bien financiero es en todo momento una buena estimación de su valor intrínseco.»

Todo lo antes expuesto, se puede interpretar así: en un mercado eficiente el precio de mercado de las acciones ${ }^{3}$ representa una buena estimación del valor real de la empresa y además, en ese mercado existe competencia entre los inversores, lo cual trae como consecuencia que cada acción refleje su precio justo, ${ }^{4}$ de acuerdo con sus características de rentabilidad y riesgo. En conclusión, los analistas suponen la inexistencia de información privilegiada en un mercado eficiente, porque ese precio reflejaría toda la información existente en el mercado, no solo los datos actuales sino también las previsiones, que son las estimaciones subjetivas que el inversionista se hace en relación a la empresa.

Además, un mercado eficiente refleja total e inmediatamente un conjunto de información en el precio de los títulos valores y es un buen indicador del valor de inversión, valor justo o valor intrínseco, que no es otra cosa que el valor presente de los comportamientos o anuncios futuros de un título o valor estimado por el analista tal como lo refieren Alexander, Sharpe y Bailey

3 También se utilizan indistintamente los términos precios bursátiles o cotizaciones bursátiles.

${ }^{4}$ La expresión Precio Justo, es quizá mal interpretada, porque todos esperan que sea un valor fijo. Realmente el precio justo es el precio de mercado, que suele ser muy variable. 
(2003). Pero, esta misma publicación supone que para que exista un mercado eficiente debería ocurrir en condiciones de un mercado perfectamente competitivo, el comportamiento racional de quienes operan en dicho mercado y las condiciones de información gratis, aunque estas utópicas condiciones sean difíciles de encontrar porque en la realidad muchas veces es costoso recolectar y procesar la información. No obstante, en un mercado donde esto cueste dinero, los analistas probablemente podrían identificar valores mal evaluados y su ganancia, al hacerlo, sería desplazada por los costes en que incurrió, según afirman Alexander, Sharpe y Bailey (2003), y el error es común en muchas publicaciones, debido a que por ejemplo se llega a pensar que si la consecución de información es demasiado costosa, los inversionistas tendrían que hacer iguales esfuerzos ante el gasto de conseguirla, por lo que deberían ganar tasas de rendimiento que ellos llegan a considerar «anormales», es decir, lo suficientemente grandes para cubrir estos gastos. Sin embargo, cuando obtenemos la cifra neta correspondiente a sus costes, su rendimiento neto es el mismo que el de los inversionistas que seleccionan sus carteras al azar, según lo señalan Weston y Copeland (1995).

Claro que el precio es la mejor estimación del valor de un bien, sobre todo financiero, pero ese precio es intensamente variable y puede cambiar de un segundo a otro en un mercado, como se afirmó antes, con la llegada de cualquier información, por lo que son las actuaciones de los individuos y las apreciaciones que ellos hacen las que alteran ese precio, que al final son juicios de valor. Pero ahí tenemos un problema de confusión entre precio y valor. Valor es lo que creemos que debería ser el precio de un determinado activo, mientras que precio es una realidad del mercado que es producto del encuentro de un comprador y un vendedor, esa realidad varía a cada momento y no necesariamente se relaciona a valoraciones fijas en el mercado.

Para estos pensadores, el mercado eficiente es aquel donde el precio de cada título es igual a su valor de inversión en todo momento, y por tanto, es imposible obtener ganancias extraordinarias, a menos que sea por casualidad o por suerte, usando este conjunto de información para componer las órdenes de compra y venta. Por ello se concluye que en un mercado eficiente, 
los inversionistas sólo obtendrán una tasa de rendimiento normal sobre sus inversiones. Esto es otro absurdo, porque supone que el mercado es de giro uniforme.

Rodríguez y Fermín (2006), señalan que si el mercado es eficiente, el pequeño inversionista estaría protegido de los efectos perjudiciales que se pudieran ocasionar por su inexperiencia sobre el funcionamiento de un mercado, porque todos los inversores especializados o no, deberían tener el mismo grado de información y por tanto, las mismas posibilidades de perder o ganar. Este es otro absurdo, por la misma razón que expusimos con los supuestos de Samuelson; la información no está igualmente distribuida.

\section{Clasificación de la Eficiencia del Mercado.}

Robert Harry en un trabajo denominado Statistical versus Clinical Prediction of the Stock Market, para el seminario de análisis del precio de los valores, que dirigía en la Universidad de Chicago en mayo de 1967, fue el primero en presentar la clasificación que universalmente se utiliza para los mercados de eficiencia fuerte, semifuerte y débil, según lo reseña el libro de Principios de Finanzas Corporativas de Brealey y Myers (1988). Fama ratificó la definición de los tres tipos de eficiencia y señaló que la noción de eficiencia de los mercados no puede ser rechazada sin un rechazo al modelo de equilibrio del mercado.

La eficiencia débil fue la primera en ser estudiada durante los años sesenta, la cual por muchas pruebas econométricas fue una teoría contrastada y aceptable. A partir de la década de los ochenta, algunos autores comenzaron a dudar de ella y ha sido tema de investigación hasta la fecha. Si el mercado es eficiente en su forma débil, los precios siguen un random walk ${ }^{5}$ o recorrido

${ }^{5}$ La fórmula más sencilla del modelo de camino aleatorio es: $Y_{t}=\mu+Y_{t}-1+\varepsilon_{t}$, donde $Y_{t}$ es una variable aleatoria cualquiera (precio), $\mu$ es el valor esperado del cambio de $Y_{t}$ y $\varepsilon_{t}$ es un elemento aleatorio que está independiente e idénticamente distribuido con media 0 y varianza $\sigma^{2}$. Sin embargo, han sido utilizados otros modelos como lo fue el modelo llamado de martingala, siendo denominada desde entonces 
aleatorio y aunque en el futuro ocurran variaciones, no se sabe cuándo se realizarán, ni cuál será su magnitud es decir, con base en los precios históricos no se puede predecir nueva información sobre el futuro.

El concepto de eficiencia semifuerte señala que el precio de las acciones refleja totalmente la información pública disponible, de naturaleza económica y financiera de la empresa y de su entorno en general, es decir precios pasados, estados financieros, las proyecciones de ganancias, las tasas de interés, el crecimiento económico, la inflación, etc. Se basa en la creencia de que todos los participantes del mercado cuentan con la misma información, por tanto, esta definición solapa a la de eficiencia débil.

Por último, la eficiencia fuerte supone que los precios bursátiles reflejan tanto la información pública disponible y conocida por todos los inversores como la información privilegiada que podrían poseer los que manejan la empresa, es decir, muestra la totalidad de la información instantáneamente, tanto pública como privada. Esta hipótesis supone que toda la información pública disponible es la histórica y la referente a los futuros planes de expansión publicitados por las empresas; si es así, no debería existir información privilegiada.

Para probar esta última forma de eficiencia, sería necesario probar que existe información privilegiada y debe haber la manera de medirla, por lo que no existe método estadístico con el cual enfrentar ese contraste. Aunque existan normativas que pretenden calificar ese tipo de actuación en el mercado. De hecho, la información privilegiada no es eficiente, si no es compartida, porque si el inversionista que posee la información «privilegiada» compra y la mantiene en secreto, eso tampoco afecta los precios y por tanto no debería sacar ventaja. Aparte de la teoría conspirativa, porque la compra enviaría una señal de «algo debe saber que no sabemos nosotros, entonces compra», Hay un problema adicional, que no se toma en cuenta en estos modelos y es el de la temporalidad de la data. Los indicadores son de cuatro tipos:

la hipótesis de martingala y que algunos consideran una condición de eficiencia de mercado y según esto, un proceso estocástico $X_{t}$ cumple con este principio cuando $E\left[X_{t+1} \mid X_{t}, X_{t+1}, \ldots\right]=X_{t} ; o$ indistintamente $E\left[X_{t+1}-X_{t} \mid X_{t}, X_{t+1}, \ldots\right]=0$. 
1) Los económicos que siempre son rezagados, es decir, todos los agregados macroeconómicos y monetarios que se producen con posterioridad al tiempo en el que son causados. El PIB se publica entre 7 y 8 semanas después que se produce el cierre de su período, de la misma manera lo único que diferencia un indicador de otro es el retraso que se produce en la información, que puede ser mayor o menor dependiendo de la metodología estadística.

2) Los financieros producidos por el mercado, que son coincidentes. Ese es el caso de los precios, tasas de interés, volúmenes de operación e índices bursátiles. Se dispone de ellos casi inmediatamente.

3) Los Contables, que al igual que los económicos, son producidos con cierto retraso. Una empresa cierra ejercicio, y con posterioridad se presentan las cuentas y los informes de gestión. Aquí hay otro problema, que es la metodología contable, que varía de acuerdo al grado de conocimiento del contador, y a las habilidades que pueda tener el gerente, como es el caso de las empresas Enron y Worldcom, por supuesto, a esto se añade los conflictos de interés que se manejen con quienes hacen la revisión de las cifras contables.

4) La información extracontable y extraeconómica. Los inversionistas, como empresarios y especuladores, observan su entorno y de él sacan señales que le permiten establecer las situaciones que se vivirán en el futuro, eso es eminentemente personal, tácito, subjetivo y no puede ser cuantificable en ningún modelo.

Como se observa hay un problema de temporalidad, los datos numerados en el punto dos, son los únicos que pueden ser utilizados para tomar decisiones rápidas en un mercado financiero. Por lo que los datos económicos y contables, mostrados en el punto 1 y 2 , serían conocidos una vez que el mercado haya reaccionado y sólo sirven para mostrarnos que nuestra predicción fue acertada, pero nunca que lo será. El punto 4 es el que más información puede suministrar, pero es el único en el que podemos darnos cuenta que no es cuantificable, el resto es igual, pero al tener valores numéricos, nos hace creer que es posible la modelización. 
La diversidad de tiempos y las metodologías hacen casi imposible la aplicación de la econometría para estimar el futuro de una inversión y menos aún de un mercado. Incluso si se usan como se hace en los modelos para estimar la eficiencia los índices de precios de las acciones, la diversa metodología de cálculo, e incluso la diversa forma de operación de un mercado en relación a otro, hace técnicamente imposible que los datos sean comparables y los resultados significativos para concluir algo cierto.

III

\section{EL MITO DE LAS MATEMÁTICAS EN LA ECONOMÍA}

La matemática es un lenguaje ideado para explicar la física como ciencia y por ello, los ingenieros la utilizan con una habilidad que escapa a los economistas. Siguiendo a Mises un economista debería aprender la mayor cantidad de disciplinas, para comprender realmente la economía, entre ellas aprender cálculo y geometría como un ingeniero, sobre todo para descifrar a cabalidad que éste no es el lenguaje apropiado para explicar los axiomas de la economía.

Pero los programas de las escuelas de economía se conforman con contratar profesores de matemáticas para dictar esta materia y son varios los errores que comenten:

a) El primero es considerar que los alumnos requieren algo de álgebra lineal, algo de cálculo diferencial y matricial, así como un poco de ecuaciones diferenciales.

b) No les enseñan geometría y tampoco les enseñan el suficiente análisis matemático para comprender lo que hacen cuando tienen una ecuación al frente.

c) Pero lo peor, no saben la suficiente teoría económica para entender que algunas variables no son biyectivas y otras son no aislables.

Si a un economista le dieran matemática como a un ingeniero, descubriría sin necesidad de leer a Say o Mises que la matemática no es el lenguaje para explicar la economía, y cuando viera las ecuaciones de los neoclásicos, comprendería que son generalizaciones 
insustentables si uno se enfrenta a la realidad, debido a que no es posible graficar una cosa como el costo de oportunidad, y que por tanto todas las ecuaciones de costo son simplificaciones que rayan en el absurdo.

Hay un ejercicio sencillo. Si usted tiene una ecuación con dos variables independientes como es el caso de la Cobb-Douglas y deriva función con la primera variable, el trabajo, eso da un valor, si deriva la segunda, da otro valor; pero ambos son consistentes con la derivada total de la ecuación. Ahora intente hacer eso con un programa econométrico y si usa las dos variables, tiene dos pendientes (derivadas), si elimina una variable y estudia sólo una, la pendiente (derivada) es distinta, si elimina la otra y usa la que antes no usó, la pendiente (derivada) vuelve a cambiar. ¿Alguien puede explicar si eso ocurre en la física?

Pero es que nuestros colegas están acostumbrados a pensar como economistas, es decir, a veces, no razonan si el computador ofrece en sus resultados un absurdo que no tiene sentido matemático. A lo sumo, alguien asomará, es un error de data, pero nadie enfrenta la realidad. Es que el paquete econométrico está dando el resultado consistente con la data que le introducimos, garbage in garbageout y como el economista tradicional está preparado para hacer toda una batería de contrastes de hipótesis, no se preocupa por la calidad de lo que le está dando el asunto. Llegándose al absurdo de afirmar que las relaciones de la teoría no se cumplen para el caso estudiado y se llega hasta el peor de los mundos que es ofrecer una teoría particular para explicar el fenómeno, es decir llegamos por las matemáticas a afirmar lo que la escuela histórica alemana hizo sin matemática.

IV

\section{LA EFICIENCIA DINÁMICA, LA ÚNICA EFICIENCIA DEL MERCADO, EN BUSCA DE UNA CONCLUSIÓN}

Para Mises (2004, p. 314) el mercado se define de esta manera:

El mercado no es ni un lugar ni una cosa ni una asociación. El mercado es un proceso puesto en marcha por las actuaciones diversas de los múltiples individuos que entre sí cooperan bajo el 
régimen de división del trabajo. Los juicios de valor de estas personas, así como las acciones que surgen de estas apreciaciones, son las fuerzas que determinan la disposición - continuamente cambiante- del mercado.

Hay que tener bien claro que mercado es donde los seres humanos interactuamos y hacemos intercambios de los bienes que producimos, distribuimos y comercializamos, por lo que no es un ente aislado de nosotros, sino nosotros mismos participamos de él y somos mercado. Destutt de Tracy (I, pp. 125-143) tiene una definición de sociedad, que es la siguiente:

La sociedad es pura y simplemente una continua serie de intercambios... ciertos animales ejecutan varios trabajos que concurren a un fin común,... pero ninguna cosa indica que ellos hagan cambios realmente... La razón de ello, en mi concepto, es que no tienen un lenguaje harto perfeccionado... y creo que esto proviene de que son incapaces de deshacer suficientemente sus ideas para generalizarlas, abstraerlas y expresarlas separada e individualmente con sus semejantes..., el hombre, por el contrario, que tiene los medios intelectuales de que carecen aquellos brutos, tiene una natural inclinación a servirse de ellas para hacer cambios; pero sí él: por lo mismo sólo el hombre tiene una verdadera sociedad; porque el comercio es toda la sociedad, como el trabajo es toda la riqueza.

Entender la Eficiencia Dinámica es entender el mercado tal cual es. Lejos de la falsa concepción de Competencia Perfecta que ofrece el Neoclasicismo, porque es técnicamente imposible rebasar las fronteras del individuo en el subjetivismo sin proceder desde una metodología deductiva y gramática, es decir, el lenguaje hablado, que es el que nos distingue de los brutos según dice Destut De Tracy y por ello, es ideal para explicar los procesos humanos, como luego afirmará Mises.

Dentro de este ámbito, como manifestación de nuestra condición humana, el lenguaje se une a la función empresarial, es decir, lo que lleva al ser humano a relacionarse en la sociedad, porque cuando actuamos empresarialmente, descubrimos y creamos oportunidades de negocio. Cada una de esas oportunidades es una nueva información que, de manera práctica, marca nuestra 
presencia en el mercado y es asimilada por el resto de los participantes. Esa información nunca es precisa y explícita, sino que es dispersa y tácita, por lo que está sujeta a la interpretación subjetiva de cada uno de los participantes. Esta realidad puede ser comentada por los economistas, pero nunca convertida en números y ecuaciones, por lo que el empresario escasamente puede convertirlo, después de ocurrido, en un ejercicio que le permita analizar sus ganancias o pérdidas, haciendo uso de la matemática financiera o registrando contablemente los detalles de su operación.

La única manera de anticipar esos resultados es en la mente, por lo que la función empresarial es especulativa, por eso prefiero la interpretación de esta palabra como derivada de Espejo (speculo), porque son los reflejos que hace el empresario del futuro, él se imagina y ve (speculari) por lo que medita y teoriza en función de lo que el anticipa lo que será el resultado de su acción. Esta acción es totalmente creativa e innovadora, por eso en los mercados financieros quienes invierten deben meditar, entender el negocio como si se fuese a fundar y hacer especulaciones, de la misma manera como el creador de un negocio cuando invente en su empresa, pero esa invención es producto de la evaluación de la información que él selecciona, clasifica, procesa y proyecta en forma de ejercicio contable. Es por ello que está lejos de realizar modelos no contables para la estimación.

Cuando actúa en el mercado transmite información, porque la acción, sea esta comprar, vender e incluso no hacer nada, comunica a los otros empresarios una información para que ellos actúen en consecuencia. Contrario a los clásicos, nuestras actuaciones no son actos aislados, producto del egoísmo solitario, sino que son influencia y son influidas por el intercambio con los otros miembros de la sociedad que conforma el mercado. Por eso, el proceso de la función empresarial es al mismo tiempo un proceso de trasmisión y coordinación, porque se puede reevaluar las posiciones al enfrentarlas a las de los demás miembros del mercado.

Pero, el mercado es como la sociedad, un proceso complejo que no tiene porque ser siempre un fenómeno sutil, puede serlo, pero también puede ser conmovedor, porque es un proceso competitivo, y es por eso que los que le tienen miedo al mercado 
creen que debe ser regulado, sin embargo, cuando se regula es cuando el mercado manifiesta de manera más violenta sus efectos competitivos y cuando más daños puede ocasionar, ejemplo de ello es la reacción del mercado a los intentos de manipulación de los bancos centrales cuando fijan las tasas para regularlos, la sobrerreacción y las crisis financieras son el resultado de este proceso que realmente buscaban suavizar el desempeño del mercado.

Por último, hay que referirse a que la función empresarial es un proceso continuo, como el mercado mismo, que no se detiene y que no puede ser detenido, los países que tenemos controles, sabemos que los mercados funcionan a la sombra de esas mismas regulaciones y que ningún tirano puede detener al individuo que se manifiesta en la sociedad que llamamos mercado.

Los mercados no son menos perfectos o más perfectos que lo que pueden ser, porque son producto de un proceso evolutivo y colectivo que es la práctica de la costumbre que impera en ellos. Es por eso que no pueden ser evaluados matemáticamente, porque no son variables homogéneas como pueden serlo un motor, para la mecánica, un corpúsculo para la biología o un compuesto para la química. El mercado, es producto de la cultura y las costumbres antropológicas de sus componentes y es cambiante, porque basta con que un nuevo individuo entre y comparta su información con los demás para hacer del mercado algo distinto a lo que fue en el instante anterior, eso no es matematizable y tampoco es comparable con otros mercados.

Pero hay dos tipos de mercado, el de los bienes que satisfacen nuestras necesidades inmediatas y el mercado de inversión, y dentro de este mercado de inversión está el mercado bursátil. La única diferencia entre ellos es la facultad que posee el mercado financiero de impedir los arbitrajes y hacerlos un proceso difícil, lo que ocasiona que los beneficios empresariales puros en un mercado financiero sean producto por un proceso de investigación, e inventiva que parece ser distinto. y por ello, somos sujetos a favorecer la creencia que puede existir tráfico de información privilegiada, eso puede ser cierto, pero cuando la información privilegiada llega de la mano de un nuevo inversionista arranca ese mismo proceso de trasmisión y coordinación que hace que 
esta información se convierta en un hecho de dominio colectivo. Si el poseedor de la información privilegiada compra, puede condicionar que su acción sea un acto autístico que probablemente no lleve a un resultado favorable para el especulador, y si lo transmite puede ser penado en las sociedades donde ésta sea una condición prohibida, o puede ser felicitado en las sociedades donde la práctica es común y bien vista; incluso puede ser execrado si, aunque no existan normas, el mercado está conformado por individuos éticos y ese comportamiento individual se riñe con la cultura de los participantes. Es ahí donde la norma puede entrar en conflicto con la realidad del mercado, porque no puede ser impuesta desde arriba, sino que es un fenómeno cataláctico, evolutivo y coordinado por la misma sociedad.

\section{REFERENCIAS BIBLIOGRÁFICAS}

Alexander, G.J., Sharpe, W.F. y Bailey, J.V. (2003): Fundamentos de Inversiones, Teoría y Práctica. Editorial Pearson Prentice Hall. 3. ${ }^{\mathrm{a}}$ ed., México.

BREALEY, R. y MYers, S., (1988): Fundamentos de Financiación Empresarial. Editorial McGraw-Hill, México, p. 297.

CoOTner, P. (1964): The Random Character of Stock Market Prices. Cambridge, M.I.T Press.

Cootner, P.H. (1962): «Stock Prices: Random Versus Systematic Changes», Industrial Management Review, vol. 3, n.․ 2, Primavera, pp. 24-45.

Destut De Tracy, A.L.C. (1824): Tratado de Economía Política, Librería de Rosa, Madrid.

DiCKEY, D.A. y FULLER, W. (1979): «Distribution of the estimators for autoregressive time series with a unit root», Journal of the American Statistical Association, n. ${ }^{\circ} 74$, pp. 427-431.

FAMA, E. (1965): «The Behavior of Stock Market Prices». Journal of Business, vol 38, Junio, pp. 34-105.

- (1976): Foundations of Finance. New York: Basic Books.

- (1970): «Efficient Capital Markets. A Review of Theory and Empirical Work», Journal of Finance, vol. 25, n.ํㅡㄹ, Mayo, pp. 383-417. 
HARRISON, B. y PATON, D. (2007): «Do fat tails matter in GARCH estimation: testing market efficiency in two transition economies». EconomicIssues, vol. 12, parte 2, pp. 15-26.

HAYeK, F.A. (1996): Precios y Producción, de F.A. Hayek Unión Editorial, Madrid..

Huerta de Soto, J. (2000): La escuela austriaca, Editorial Síntesis, Madrid.

- (2001): Socialismo, Cálculo Económico y Función Empresarial, 2. ${ }^{\mathrm{a}}$ ed., Unión Editorial, Madrid.

JACQUILLAT, B. y SolniK, B. (1975): Mercados Financieros y Gestión de Carteras de Valores, Editorial Tecniban S.A., Madrid, p. 22.

Kendall, M. (1953): «The Analysis of Economics Times Series». Part I. Journal of Royal Statical Society, 96, pp. 11-25.

Keynes, J.M. (1981): Teoría General de la Ocupación, el Interés y el Dinero, Fondo de Cultura Económica. Bogotá.

KIRZNER, I.M.(1995): Creatividad, Capitalismo y Justicia Distributiva, Unión Editorial, Madrid.

- (1992): The Meaning of Market Process. Essays in the Development of Modern Austrian Economics, Routledge, Chapman \& Hall, London.

- (1998): Competencia y Empresarialidad, Unión Editorial Madrid. Kyrtsou, C.; LeONTITSIS, A. y Siriopoulos, C. (2006): «Exploring the impacto of calendar effects on the dynamic structure and forecasts of financial time series». International Journal of Theoretical and Applied Finance, vol. 9, n.. 1, pp. 1-22.

LI, H. y Rosser, J.B. Jr. (2004): «Market dynamics and stock price volatility». The European Physical Journal B. 39, pp. 409-413.

LJung, G.M. y Box, G.E.P. (1978): «On a Measure of a Lack of Fit in Time Series Models». Biometrika 65. pp. 297-303.

MANDELBRot, B. (1963): «The Variation of Certain Speculative Prices». Journal of Busines, n. ${ }^{\circ} 36$, octubre, pp. 394-419.

MARTínez, C. (2006): La Teoría Evolutiva de las Instituciones. Unión Editorial Madrid.

Menger, C. (2006): El Método de las Ciencias Sociales. Unión Editorial, Madrid.

MirandA, T.B. y BLASS, S.R. (2006): «Persistence and mean reversión: analyzing sector indices for Brazil». Economía Aplicada, vol. 10, n.. 2. Ribeirao Preto. Junio, pp. 1-13. 
Mises, L. von (2004): La Acción Humana, tratado de economía política, 7. ${ }^{\mathrm{a}}$ ed., Unión Editorial, Madrid.

Osborne, M. (1962): «Periodic Structure in the Brownian Motion in The Stock Prices», 1962, Operations Research, vol. 10, n. ${ }^{\circ}$ 3, pp. 345-379.

Osborne, M.F. (1959): «Brownian Motion in the Stock Market». Operation Research, vol. 7, marzo-abril, pp. 145-173.

Phillips, P.C.B y Perron (1988): «Testing for a Unit Root in Time Series Regression», Biometrika, n.․ 75, pp. 335-346.

RodrígueZ, L.M. y FERMín, J.S. (2006): «Mercado eficiente y caminata aleatoria en la Bolsa de Valores de Caracas». Asociación Interciencia, Caracas Venezuela, diciembre, año/vol. 31, n. ${ }^{\mathrm{o}}$ 012, pp. 888-893.

RÖPKe, W. (1996): Más allá de la Oferta y la Demanda, Unión Editorial, Madrid.

SAmpaio, G.A.; SARto, J.L.; BARbosa, A.; Sigueira, I. y Corrar, L.J. (2006): «El estudio del anuncio de la adhesión a los niveles diferenciados de gobierno corporativo con la utilización de estudio del evento».VI Conferencia Internacional de Finanzas, Santiago mayo de 2006, p. 95.

Samuelson, P. (1965): «Proof that Properly Anticipated Prices Fluctuate Randomly». Industrial Management Review, vol. 6, pp. 41-49.

SÁNCHEZ, J.L. (2007): Curso de bolsa y mercados financieros, Instituto Español de Analistas Financieros. 4. a edición actualizada. Editorial Ariel S.A. Barcelona, España, p. 541.

Swoboda, C. (2001): La hipótesis del Random Walk en el Mercado de Valores de la Argentina. Período 1992-2000. http:/ / www.eco. unc.edu.ar/organizacion/departamentos/economia/doc de_trabajo_n17_(swoboda).doc. descargado el : 14 de abril de 2012.

Weston, J. y Copeland, T. (1995): Finanzas en Administración, 9. a ed., vol. I, Editorial McGraw-Hill, pp. 111-116.

Zhang, T., LI, J. y Malone, P. (2004): «Closed-End Fund Discounts in Chinese Stock Markets». The Chinese Economy, vol. 37, n. ${ }^{\circ}$, pp. 17-38. 